\title{
DIAGNÓSTICOS DE ENFERMAGEM IDENTIFICADOS EM IDOSOS ATENDIDOS EM UM SERVIÇO DE RADIOTERAPIA
}

\author{
NURSING DIAGNOSES IDENTIFIED IN ELDERLY PATIENTS SEEN AT A \\ RADIOTHERAPY SERVICE
}

\section{DIAGNÓSTICOS DE ENFERMERÍA IDENTIFICADOS EN ANCIANOS ATENDIDOS EN UN SERVICIO DE RADIOTERAPIA}

Cláudia Mendes da Silva ${ }^{1}$, Amanda Rocha da Silva França², Elizabeth Moura Soares de Souza ${ }^{3}$, Maria Socorro da Silva Paiva Cavalcante ${ }^{4}$, Maria da Glória de Lima Bomfim ${ }^{5}$

\begin{abstract}
RESUMO
Objetivo: este trabalho teve como objetivo identificar os diagnósticos de Enfermagem apresentados por pacientes idosos submetidos à radioterapia. Método: trata-se de um estudo quantitativo e descritivo. A coleta de dados ocorreu por meio do levantamento de dados secundários provenientes dos registros de Enfermagem presentes no prontuário eletrônico do sistema MV/PEP/HUPAA. A amostra foi restrita a informações relativas à consulta de Enfermagem a pacientes idosos em tratamento radioterápico, sendo selecionados 22 prontuários. Resultados: identificaram-se 123 diagnósticos de Enfermagem onde $100 \%$ dos idosos apresentaram o diagnóstico de risco de integridade da pele prejudicada. Conclusão: o estudo oportunizou compreender a relevância do processo de Enfermagem e evidenciou que o uso dos diagnósticos de Enfermagem pode propiciar a autonomia do enfermeiro.
\end{abstract}

Descritores: Processo de Enfermagem; Idoso; Oncologia; Radioterapia.

\begin{abstract}
Objective: this study aimed to identify nursing diagnoses presented by elderly patients submitted to radiotherapy. Method: it is a quantitative and descriptive study. Data collection was done through the collection of secondary data from the Nursing records present in the electronic medical record of the MV / PEP / HUPAA system. The sample was restricted to information related to the Nursing consultation of elderly patients in radiotherapy, with 22 medical records being selected. Results: 123 Nursing diagnoses were identified in which $100 \%$ of the elderly presented a diagnosis of impaired skin integrity risk. Conclusion: the study facilitated the understanding of the relevance of the
\end{abstract}

1,2,4,5 Especialistas. Universidade Federal de Alagoas/UFAL. Maceió (AL), Brasil.

${ }^{3}$ Doutora. Universidade Federal de Alagoas/UFAL. Maceió (AL), Brasil.

Rev. Port. Saúde e Sociedade. 2019;4(1): 1018 - 1027. 
Nursing process and showed that the use of Nursing diagnoses can provide nurses autonomy.

\section{Descriptors: Nursing Process; Aged; Medical Oncology; Radiotherapy.}

\section{RESUMEN}

Objetivo: este trabajo tuvo como objetivo identificar los diagnósticos de Enfermería presentados por pacientes ancianos sometidos a la radioterapia. Método: se trata de un estudio cuantitativo y descriptivo. La recolección de datos ocurrió por medio del levantamiento de datos secundarios provenientes de los registros de Enfermería presentes en el prontuario electrónico del sistema MV / PEP / HUPAA. La muestra fue restringida a informaciones relativas a la consulta de Enfermería a pacientes ancianos en tratamiento radioterápico, siendo seleccionados 22 prontuarios. Resultados: se identificaron 123 diagnósticos de Enfermería, donde el 100\% de los ancianos presentaron el diagnóstico de riesgo de integridad de la piel perjudicada. Conclusión: el estudio proporcionó comprender la relevancia del proceso de Enfermería y evidenció que el uso de los diagnósticos de Enfermería puede propiciar la autonomía del enfermero.

\section{Descriptores: Proceso de Enfermería; Anciano; Oncología Médica; Radioterapia.}

\section{INTRODUÇÃO}

À medida que a população mundial envelhece, os agravos decorrentes do processo de envelhecimento passam a ser identificados. Dentre esses agravos, inclui-se o avanço das doenças crônicas. Estima-se que, em 2020, essas patologias representarão $80 \%$ dos problemas de saúde, incluindo as neoplasias que possuem maior incidência em populações acima dos 60 anos. ${ }^{1}$

A Organização Mundial de Saúde (OMS) estima, para o ano de 2025, cerca de 20 milhões de casos novos de câncer. No Brasil, dados apontaram, para o biênio 2016-2017, um total de 600 mil casos novos previstos, reforçando a magnitude dessa doença como problema de saúde pública. ${ }^{2}$

Em Alagoas, a estimativa foi de 4.680 casos novos de câncer para o biênio 2016-2017, representando uma taxa bruta de 281,5 casos por 100 mil habitantes. Esta patologia é referida como a segunda maior causa de morte, ficando abaixo apenas das doenças cardiovasculares. ${ }^{2}$

Diante do exposto, considerando o panorama epidemiológico de neoplasias na população e as diversas linhas terapêuticas existentes na atualidade, destacase a radioterapia, um tratamento local que utiliza a radiação ionizante produzida por aparelhos ou emitida por radioisótopos naturais para impedir a multiplicação celular ou induzir a morte por apoptose das células neoplásicas. ${ }^{3}$ No entanto, apesar de seus benefícios no tratamento do câncer, as radiações podem acarretar manifestações de toxicidade para o indivíduo. Faz-se necessária, assim,

Rev. Port. Saúde e Sociedade. 2019;4(1): 1018 - 1027. 
a inclusão de medidas de prevenção a fim de minimizar a radiotoxicidade durante o tratamento. ${ }^{1}$

Um estudo voltado à avaliação da adesão dos pacientes às orientações fornecidas na consulta de Enfermagem em radioterapia demonstrou que a maioria dos pacientes (54\%) que aderiram às orientações de autocuidado não desenvolveu radiodermites. ${ }^{4}$ Dessa forma, a consulta de Enfermagem, no setor de radioterapia, merece especial enfoque, pois é a atividade mais específica exercida pelo enfermeiro no setor, sendo ferramenta essencial para a qualidade de vida do paciente e condição favorável para se ofertar um cuidado seguro. ${ }^{3}$

O Art. 11, inciso I, alínea "i", da Lei no 7.498, de 25 de junho de 1986,5 regulamenta e legitima a Consulta de Enfermagem como sendo uma atividade privativa do enfermeiro, a qual utiliza componentes do método científico para identificar situações de saúde/doença, bem como prescrever e colocar em prática ações de Enfermagem que contribuam para a promoção da saúde, prevenção e proteção de agravos, recuperação e reabilitação do indivíduo, família e comunidade.

A Sistematização da Assistência de Enfermagem, caracterizada como um método pelo qual o enfermeiro organiza suas ações e direciona o cuidado, constitui um importante embasamento para o estabelecimento do Processo de Enfermagem. É por meio desse processo que as necessidades do paciente são identificadas e um plano de cuidado é traçado para que possa ser executado pela equipe de Enfermagem com o objetivo de proporcionar o seu bem-estar. ${ }^{6}$

Nesse sentido, a Enfermagem dispõe de diversos sistemas de classificação, destacando-se a Classificação Internacional para a Prática de Enfermagem CIPE, um instrumento tecnológico que, no decorrer da execução do Processo de Enfermagem, auxilia o raciocínio e a tomada de decisão clínica; proporciona a comunicação entre os profissionais de Enfermagem e de outras áreas e facilita a documentação da prática profissional, fato fundamental tanto para a avaliação quanto para a visibilidade da contribuição da Enfermagem à saúde das pessoas, famílias e coletividades humanas. 7,8

Para tanto, em consequência da toxicidade do tratamento com radiação ionizante aos pacientes oncológicos e da relevância da sistematização da assistência de Enfermagem em radioterapia, surgiu a seguinte pergunta norteadora: "Quais os diagnósticos de Enfermagem presentes nos registros de Enfermagem de pacientes idosos atendidos em um serviço de radioterapia?".

Rev. Port. Saúde e Sociedade. 2019;4(1): 1018 - 1027. 
Para responder a essa questão, o estudo teve como objetivo identificar os diagnósticos de Enfermagem de pacientes idosos atendidos em um serviço de radioterapia.

\section{MÉTODO}

Estudo quantitativo e descritivo desenvolvido durante o Programa de Residência Multiprofissional em Saúde do Adulto e do Idoso da Universidade Federal de Alagoas, em um período de prática no setor de radioterapia.

A pesquisa foi realizada em um hospital universitário localizado no município de Maceió-AL, pertencente à rede pública, de níveis secundário e terciário de atenção à saúde, no Setor de Radioterapia do Centro de Assistência de Alta Complexidade em Oncologia - CACON, no qual se procuraram identificar os diagnósticos de Enfermagem apresentados por pacientes idosos acima de 60 anos no período de agosto de 2017.

A amostra foi restrita a informações relativas à consulta de Enfermagem a pacientes idosos em tratamento radioterápico, tendo como critérios de inclusão os prontuários de pessoas acima de 60 anos, que se encontrassem em radioterapia durante o mês de agosto de 2017; e como critérios de exclusão aqueles prontuários que não apresentassem registros de Enfermagem. Assim, foram selecionados 22 prontuários de idosos de um total de 40 prontuários.

A coleta de dados deu-se por meio de levantamento de dados secundários provenientes dos registros de Enfermagem presentes no prontuário eletrônico do sistema MV/PEP/HUPAA. Os números obtidos foram submetidos à analise descritiva, por meio de frequências absoluta e relativa ( $\mathrm{N}$ e \%) e, posteriormente, compilados e tabulados no software Microsoft Excel 2016 for Windows $®$.

O estudo foi aprovado pelo Comitê de Ética em Pesquisa da Universidade Federal de Alagoas, sob o número de protocolo 2.682.221, conforme a Resolução n. 0 466/12, do Conselho Nacional de Saúde, para pesquisas com seres humanos.

\section{RESULTADOS}

Ao considerar o quantitativo de pacientes da pesquisa atendidos no serviço de radioterapia do HUPAA, $72,7 \%$ desses eram do sexo feminino, com faixa

Rev. Port. Saúde e Sociedade. 2019;4(1): 1018 - 1027. 
etária variando entre 60 e 106 anos de idade e mediana de 70 anos. Quanto ao tempo de tratamento, 54,5\% realizaram o tratamento em cinco semanas; $18,2 \%$, em até quatro semanas; $13,6 \%$, em seis semanas e $13,6 \%$, em sete semanas ou mais.

Em relação aos diagnósticos médicos no período estudado, foi possível observar que o câncer ginecológico ocupou o primeiro lugar no percentual de casos, apresentando 27,3\%, seguido dos cânceres de mama e de esôfago, responsáveis por $18,2 \%$ cada. Os cânceres de cabeça e pescoço representaram um valor de 9,1\%, assim como o câncer de reto/ânus. Enquanto os 18,5\% restantes foram referentes a câncer de próstata, câncer de sistema nervoso central, câncer de pele e câncer de pulmão, computando um caso para cada diagnóstico (Tabela 1).

\section{Tabela 1. Diagnósticos médicos de pacientes atendidos no serviço de radioterapia do CACON/HUPAA.}

\begin{tabular}{|c|c|c|}
\hline Diagnósticos Médicos & $\mathbf{N *}$ & $\% * *$ \\
\hline Câncer ginecológico & 6 & 27,3 \\
\hline Câncer de esôfago & 4 & 18,2 \\
\hline Câncer de mama & 4 & 18,2 \\
\hline Câncer de cabeça e pescoço & 2 & 9,1 \\
\hline Câncer de reto/ânus & 2 & 9,1 \\
\hline Câncer de próstata & 1 & 4,5 \\
\hline Câncer de sistema nervoso central & 1 & 4,5 \\
\hline Câncer de pele & 1 & 4,5 \\
\hline Câncer de pulmão & 1 & 4,5 \\
\hline Total & 22 & 100 \\
\hline
\end{tabular}

Fonte: PEP de um HU. Legenda: *frequência absoluta; **frequência relativa.

$\mathrm{Na}$ tabela 2, têm-se os Diagnósticos de Enfermagem identificados em pacientes idosos oncológicos submetidos à radioterapia, segundo a CIPE 2015. O total de diagnósticos e a porcentagem com relação ao seu predomínio são apresentados. Destaca-se a presença de 123 diagnósticos de Enfermagem identificados nesses pacientes, totalizando uma média de seis diagnósticos por indivíduo.

É possível averiguar que $100 \%$ dos idosos apresentaram o diagnóstico de "risco de integridade da pele prejudicada". Enquanto os diagnósticos de "falta de conhecimento sobre radioterapia" e "integridade da pele prejudicada" ocuparam o segundo e terceiro lugar, com $86,4 \%$ e $45,5 \%$, respectivamente, no período

Rev. Port. Saúde e Sociedade. 2019;4(1): 1018 - 1027. 
estudado. Infere-se que aqueles diagnósticos com menor frequência podem estar associados a particularidades do indivíduo e de sua doença.

Tabela 2. Diagnósticos de Enfermagem de pacientes atendidos no serviço de radioterapia do CACON/HUPAA.

\begin{tabular}{|c|c|c|}
\hline Diagnósticos de Enfermagem & $\mathbf{N} *$ & $\% * *$ \\
\hline Risco de integridade da pele prejudicada & 22 & 100 \\
\hline Falta de conhecimento sobre radioterapia & 19 & 86,4 \\
\hline Integridade da pele prejudicada & 10 & 45,5 \\
\hline Padrão de ingestão de líquidos prejudicado & 7 & 31,8 \\
\hline Dor & 4 & 18,2 \\
\hline Náusea & 4 & 18,2 \\
\hline Ferida presente & 3 & 13,6 \\
\hline Padrão de ingestão de alimentos prejudicado & 3 & 13,6 \\
\hline Apetite prejudicado & 3 & 13,6 \\
\hline Dor abdominal & 3 & 13,6 \\
\hline Ingestão de líquidos prejudicada & 3 & 13,6 \\
\hline Ingestão de alimentos insuficiente & 3 & 13,6 \\
\hline Conhecimento sobre radioterapia baixo & 3 & 13,6 \\
\hline Constipação & 2 & 9,1 \\
\hline Diarreia & 2 & 9,1 \\
\hline Padrão de ingestão de líquidos melhorado & 2 & 9,1 \\
\hline Alimentação, por si próprio, prejudicada & 2 & 9,1 \\
\hline Sono prejudicado & 2 & 9,1 \\
\hline Pressão arterial alterada & 2 & 9,1 \\
\hline Incontinência urinária & 1 & 4,5 \\
\hline Mobilidade do braço direito prejudicada & 1 & 4,5 \\
\hline Padrão de higiene oral prejudicado & 1 & 4,5 \\
\hline Edema periférico & 1 & 4,5 \\
\hline Risco de queda & 1 & 4,5 \\
\hline Autocuidado eficaz & 1 & 4,5 \\
\hline Risco de desidratação & 1 & 4,5 \\
\hline Disúria & 1 & 4,5 \\
\hline Membrana mucosa oral seca & 1 & 4,5 \\
\hline Presença de tubo enteral & 1 & 4,5 \\
\hline Constipação presente & 1 & 4,5 \\
\hline Aceitação do estado de saúde prejudicada & 1 & 4,5 \\
\hline Não adesão ao regime de líquidos & 1 & 4,5 \\
\hline Peso prejudicado & 1 & 4,5 \\
\hline Baixa autoestima & 1 & 4,5 \\
\hline Padrão de ingestão de alimentos eficaz & 1 & 4,5 \\
\hline Função do sistema urinário prejudicado & 1 & 4,5 \\
\hline Paladar prejudicado & 1 & 4,5 \\
\hline Deficit de autocuidado & 1 & 4,5 \\
\hline Tabagismo prévio & 1 & 4,5 \\
\hline Prurido presente & 1 & 4,5 \\
\hline
\end{tabular}

Rev. Port. Saúde e Sociedade. 2019;4(1): 1018 - 1027. 
Fonte: PEP de um HU. Legenda: *frequência absoluta; ${ }^{*}$ frequência relativa.

\section{DISCUSSÃO}

As reações agudas de pele, também conhecidas como radiodermites, constituem um dos efeitos mais comuns em pacientes submetidos à radioterapia, as quais foram representadas pelos diagnósticos "risco de integridade da pele prejudicada", "integridade da pele prejudicada" e "ferida presente". Essas reações podem variar de um leve eritema com prurido até a necrose tecidual, passando por descamação seca ou úmida. ${ }^{9}$

Em relação a estes diagnósticos, as medidas de prevenção são primordiais e precisam ser prescritas durante a consulta de Enfermagem com o intuito de diminuir a radiotoxicidade. ${ }^{4}$ Dentre as intervenções implementadas pelo enfermeiro, destacam-se as orientações quanto aos cuidados com a pele, no que se refere à hidratação, à não exposição solar, higiene e alimentação. Além disso, inclui-se o uso de gel à base de aloe vera durante todo o tratamento que, tem a finalidade de impedir a ocorrência e/ou o aumento do grau de radiodermite, bem como amenizar os efeitos da radiação na pele. ${ }^{4}$

Em se tratando dos diagnósticos "falta de conhecimento sobre radioterapia" e "conhecimento sobre radioterapia baixo", a consulta de Enfermagem elucida dúvidas comuns e isoladas de pacientes que iniciam o processo terapêutico, constituindo-se como um momento oportuno para o melhor entendimento do paciente sobre a doença e a importância de adesão às sessões de radioterapia. ${ }^{4,10}$

Nesse momento, a consulta de Enfermagem propicia, ao idoso, informações sobre a radioterapia, as possíveis reações adversas e o seu manejo, bem como a importância do prosseguimento do tratamento. Ao mesmo tempo, este usuário é estimulado a expor suas dúvidas e anseios sobre o processo terapêutico.

A baixa ingestão de alimentos e líquidos pelos idosos, observada e evidenciada por meio dos diagnósticos de "ingestão de líquidos ou alimentos prejudicada/insuficiente", "padrão de ingestão de líquidos ou alimentos prejudicado" e "risco de desidratação", instrumentaliza o enfermeiro na consulta

Rev. Port. Saúde e Sociedade. 2019;4(1): 1018 - 1027. 
de Enfermagem a enfatizar a adesão a uma dieta apropriada e à ingestão de líquidos adequada. ${ }^{4}$

O diagnóstico de Enfermagem "membrana mucosa oral seca" relaciona-se à mucosite, que é uma reação inflamatória da mucosa oral caracterizada por eritema e edema na mucosa seguidos por comumente ulceração e descamação. A radiação, quando em doses entre 40 e 65 Gy, pode ocasionar reação inflamatória degenerativa, especialmente das células serosas das glândulas salivares, provocando uma diminuição do fluxo salivar e, consequentemente, a xerostomia. ${ }^{3}$

As intervenções para a prevenção e o tratamento da mucosite oral em radioterapia são de suma importância na consulta de Enfermagem, onde o enfermeiro orienta a prática de higiene oral como uma estratégia que reduz o desenvolvimento de micro-organismos, diminuindo o risco da mucosite severa. ${ }^{11}$

Além dos diagnósticos citados acima, reações relacionadas à dor, à mobilidade e a sensações alteradas no lado afetado, fadiga, perda da autoestima, ansiedade, medo e sentimento de isolamento estão também associadas à radioterapia. ${ }^{9}$

Os sintomas do tratamento do câncer são uma das maiores dificuldades enfrentadas pelos pacientes idosos durante as sessões de radioterapia. Esta, associada à doença, resulta em uma redução no nível de desempenho funcional do idoso e aumento da depressão, o que altera a sua qualidade de vida e, consequentemente, a sua percepção em relação ao seu estado geral de saúde. ${ }^{10}$

Observa-se que, para os pacientes oncológicos, em especial, os idosos, conviver com o câncer pode provocar sofrimento. Acredita-se que esse sentimento pode estar relacionado às suas perspectivas de vida, crenças e temores relacionados à doença e ao tratamento, fato que denota a importância do desenvolvimento de cuidados de Enfermagem que os auxiliem durante esse enfrentamento. ${ }^{9}$

Diante do exposto, auxiliar o paciente e familiares a entender o processo em que estão inseridos é tornar o indivíduo capaz de enfrentar este momento de sua vida, apoiando-o para as possíveis complicações. É primordial uma comunicação mais efetiva entre a equipe e o paciente, dado que poderá favorecer a adesão ao tratamento e aos cuidados a serem implementados, bem como contribuir para minimizar os riscos da estratégia terapêutica. ${ }^{12}$ Para tanto, verifica-se que o processo de Enfermagem é um instrumento essencial para a

Rev. Port. Saúde e Sociedade. 2019;4(1): 1018 - 1027. 
organização da prática clínica e a CIPE é uma classificação que utiliza métodos práticos para a elaboração do diagnóstico e seleção das intervenções que facilitam a sistematização da assistência de Enfermagem. ${ }^{12}$

\section{CONCLUSÃO}

A experiência com idosos no setor de radioterapia do CACON oportunizou compreender a importância do processo de Enfermagem na atenção a esses indivíduos, por ser uma ferramenta útil que o enfermeiro utiliza para sistematizar o cuidado visando a melhorar o estado de saúde e a qualidade de vida desses pacientes. Ao mesmo tempo, foi possível evidenciar, por meio dos resultados deste relato, que o uso dos diagnósticos de Enfermagem propicia a autonomia do enfermeiro, uma vez que é a base para o desenvolvimento das intervenções de Enfermagem, oportunizando, portanto, o exercício do raciocínio crítico e o julgamento clínico deste profissional.

\section{REFERENCES}

1. Marinho MD, Pontes KLC, Alves MJOC, Lima FA, Araújo CRF. Perfil dos idosos portadores de câncer atendidos pela Fundação Assistencial da Paraíba (FAP) em tratamento de radio e quimioterapia. In: 40 Congresso Internacional Envelhecimento Humano. Anais do 40 Congresso Internacional Envelhecimento Humano. 2015 [Internet]. Campina Grande: CIEH; 2015 [cited 2018 Aug 21]; 2(1):1-6. Available from: http://docplayer.com.br/41787034-Perfil-dos-idososportadores-de-cancer-atendidos-pela-fundacao-assistencial-da-paraiba-fap-emtratamento-de-radio-e-quimioterapia.html

2. Ministério da Saúde (BR), Instituto Nacional do Câncer José Alencar Gomes da Silva. Estimativa 2016: incidência de câncer no Brasil [Internet]. Rio de Janeiro: Ministério da Saúde; 2015 [cited 2017 Aug 22]. Available from: http://www.inca.gov.br/bvscontrolecancer/publicacoes/edicao/Estimativa_2016.p df.

3. Leite FMC, Ferreira FM, Cruz MAS, Lima EFA, Primo CC. Nursing diagnosis related to the adverse effects of radiotherapy. Rev Min Enferm. 2013 Oct/Dec; 17(4):946-51. Doi: http://www.dx.doi.org/10.5935/1415-2762.20130068

4. Andrade KBS, Francz ACL, Grellmann MS, Belchior PC, Oliveira JA, Wassita DN. Nursing consultation: evaluation of adherence to self-care in patients undergoing radiotherapy. Rev Enferm UERJ. 2014; 22(5):622-28. Doi: http://dx.doi.org/10.12957/reuerj.2014.11227

5. Lei no 7.498 , de 25 de junho de 1986. Dispõe sobre a regulamentação do exercício da enfermagem, e dá outras providências. Diário Oficial da União [Internet]. 1986 [cited 2018 Sept 10]. Available from: http://www.cofen.gov.br/lei-n-749886-de-25-de-junho-de-1986_4161.html

Rev. Port. Saúde e Sociedade. 2019;4(1): 1018 - 1027. 
6. Lima APS, Chianca TCM, Tannure MC. Assessment of nursing care using indicators generated by software. Rev Latino-Am Enfermagem. 2015 Mar/Apr; 23(2):23441. Doi: http://dx.doi.org/10.1590/0104-1169.0177.2547

7. Conselho Internacional de Enfermeiros. Classificação Internacional para a Prática de Enfermagem: versão 2.0 [Internet]. São Paulo: Algol; 2011 [cited 2017 Aug 21]. Available from: https://www.travessa.com.br/cipe-versao-2-classificacaointernacional-para-a-pratica-de-enfermagem-versao-2-0/artigo/0a0067af-9ff44d55-ad5e-1d8d7f2f1143

8. Garcia TR, Coenen AM, Bartz CC. Classificação Internacional para a Prática de Enfermagem (CIPE®) [Internet]. Porto Alegre: Artmed; 2016 [cited 2017 Aug 23]. Available from: http://srvd.grupoa.com.br/uploads/imagensExtra/legado/G/GARCIA_Telma_Ribeir o/CIPE_2017/Lib/Amostra.pdf

9. Martins MS, Marta CB, Silva PO, Siqueira APR, Gallasch CH, Peregrino AAF. Nursing Consultation in the Head and Neck Cancer Radiotherapy: a Cost-Health Utility Relationship Analysis. J res fundam care online. 2018 July/Sept; 10(3):74652. Doi: http://dx.doi.org/10.9789/2175-5361.2018.v10i3.746-752

10. Toneli BF, Paula JM, Nicolussi AC, Sawada NO. Health-related quality of life of the elderly with cancer in adjuvant treatment. Rev Rene. 2014 Nov/Dec; 15(6):103038. Doi: $10.15253 / 2175-6783.2014000600017$.

11. Albuquerque ILS, Camargo TC. Prevenção e tratamento da mucosite oral induzida por radioterapia: revisão da literatura. Rev Bras Cancerol [Internet]. 2007 Apr/June [cited 2018 Aug 7]; 53(2):195-209. Available from: http://bases.bireme.br/cgi-

bin/wxislind.exe/iah/online/?IsisScript=iah/iah.xis\&src=google\&base=LILACS\&lan $\mathrm{g}=\mathrm{p} \&$ nextAction $=$ Ink\&exprSearch $=523363 \&$ indexSearch $=$ ID

12. Cavalari E, Nogueira MS, Fava SMCL, Cesarino CB, Martin JFV. Adherence to treatment: a study with hypertensive outpatients. Rev Enferm UER] [Internet]. 2012 Jan/Mar [cited 2017 Aug 25]; 20:67-72. Available from: https://www.epublicacoes.uerj.br/index.php/enfermagemuerj/article/view/3979/2761

Rev. Port. Saúde e Sociedade. 2019;4(1): 1018 - 1027. 